\title{
OPTIMAL SENSOR POSITIONING ON PRESSURIZED EQUIPMENT BASED ON VALUE OF INFORMATION
}

\author{
Seyed Mojtaba Hoseyni ${ }^{1}$, Francesco Di Maio ${ }^{1}$, Enrico Zio ${ }^{1,2,3}$ \\ ${ }^{1}$ Energy Department, Politecnico di Milano, Via La Masa 34, 20156 Milano, Italy \\ ${ }^{2}$ MINES ParisTech / PSL Université Paris, Centre de Recherche sur les Risques et les Crises (CRC), Sophia \\ Antipolis, France \\ ${ }^{3}$ Eminent Scholar, Department of Nuclear Engineering, Kyung Hee University
}

\begin{abstract}
In this work, we apply a simulation-based framework that makes use of the Value of Information (VoI) for identifying the optimal spatial positioning of sensors on pressurized equipment. VoI is a utility-based Figure of Merit (FoM) which quantifies the benefits/losses of acquiring information. Sensors are typically positioned on pressurized equipment in line with specific recommendations based on operational experience, like UNI 11096 in Italy. We show that the recommendations in UNI 11096 are, indeed, justified and that, incidentally, relying on VoI for the optimization of the sensor positioning, one can achieve the same monitoring performance, as measured by VoI, where following UNI 11096, but with a reduced number of sensors. The proposed VoI-based approach can, thus, be used to confirm or revise recommendations coming from operational experience.
\end{abstract}

Keywords: Value of Information (VoI), Optimization, Sensors Positioning, Bayesian Statistics, Pressure Vessel, Creep. 


\section{Acronyms}

$\begin{array}{ll}\text { FoM } & \text { Figure of Merit } \\ \text { GP } & \text { Gaussian Process } \\ \text { NDT } & \text { Non-Destructive Test } \\ \text { PFBR } & \text { Prototype Fast Breeder Reactor } \\ \text { SG } & \text { Steam Generator } \\ \text { SSC } & \text { Systems, Structures, and Components } \\ \text { UTS } & \text { Ultrasonic Thickness Testing } \\ \text { VoI } & \text { Value of Information }\end{array}$

\section{Nomenclature}

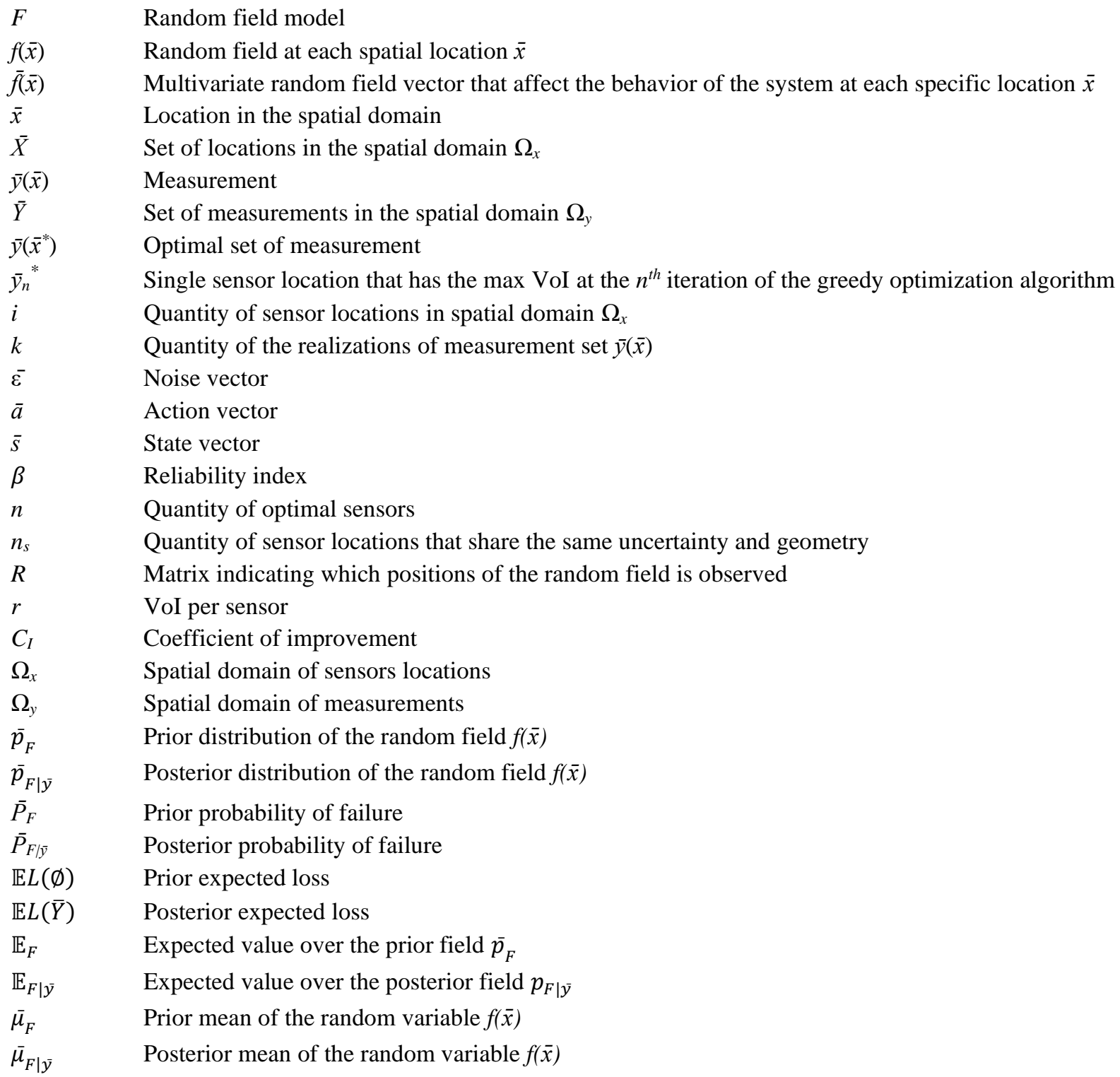




$\begin{array}{ll}\bar{\mu}_{\bar{Y}} & \text { Mean of the random variable } f(\bar{x}) \text { derived from the measurement set } \\ \mu_{g} & \text { Mean of the limit state } \\ \sigma_{g} & \text { Standard deviation of the limit state } \\ \Sigma_{F} & \text { Prior covariance matrix of the random variable } f(\bar{x}) \\ \Sigma_{F \mid \bar{Y}} & \text { Posterior covariance matrix of the random variable } f(\bar{x}) \\ \Sigma_{\bar{Y}} & \text { Covariance matrix of the measurement } \\ \Sigma_{\varepsilon} & \text { Covariance matrix of the noise } \\ C_{f} & \text { Cost of failure } \\ C_{p} & \text { Cost of failure prevention } \\ V o I_{U N I} & \text { VoI obtained by UNI 11096 sensor positioning } \\ n_{U N I} & \text { Number of sensors as for UNI 11096 sensor positioning } \\ r_{U N I} & \text { VoI ratio for UNI 11096 sensor positioning } \\ C & \text { Cost of measurement } \\ V o I & \text { Value of information } \\ M & \text { Benefit of conducting measurement } \\ f_{s}(\bar{x}) & \text { Strength random field } \\ f_{t}(\bar{x}) & \text { Failure threshold field } \\ g(\bar{x}) & \text { Limit state function }\end{array}$

\section{INTRODUCTION}

Safety-critical Systems, Structures, and Components (SSCs) need to comply with safety standards. Guidelines are developed for testing the compliance to the safety standards. However, the guidelines are not necessarily resulting from a formal process and may involve shortcomings and over-conservatism which may lead to unnecessary costs $(1,2)$. Also, guidelines should not slow down industrial advancements but rather flexibly reflect methodological developments and technological advancements (3). For example, let us consider the Italian regulatory code UNI 11096 (4), the European norm EN 13445 (5), or the American norm ASME Boiler \& Pressure Vessel Code (BPVC) (6), all aimed at describing the positioning of thickness sensors to monitor pressurized equipment that may suffer of creep, and all based on experimental/operational past experience, such as in-service inspections and structural analyses (7). These guidelines might be challenged when dealing with new SSCs on which there is limited experience.

In the context of SSCs creep, for example, market competition has pushed industry to operate the SSCs at high design stresses (and, in some cases, beyond design stresses) $(8,9)$, while new knowledge on creep phenomenon becomes available. This has recently resulted in a deep revision of the guidelines $(10,11)$, 
making scheduled inspections by Non-Destructive Tests (NDTs) recommended to prevent SSCs creep and failures $(12,13,1,14)$. On the other hand, condition monitoring and intelligent analysis of data collected by sensors may help predicting degradation escalation and anticipating the risk of failure $(15,16,17,18,19)$.

In this work, we use the concept of Value of Information (VoI) to find how to optimally position ultrasonic thickness gauges on a pressurized equipment, for largest benefit in terms of reduced costs and increased data accuracy. VoI is a mathematical concept standing on Bayesian statistical decision theory and applied in different fields for supporting decision making, from economics to engineering (20). For practical meaning, we consider the problem of positioning sensors on a simulated manifold of a Steam Generator (SG). The novelty of this work lies in use of the VoI-based sensors positioning framework for comparing the outcomes with standards/recommendations/guidelines for monitoring of energy SSCs issued by regulatory bodies, to confirm their validity or suggest improvements. Results show that the VoI based sensor positioning allows reducing the number of sensors to be positioned with respect to the guidelines in (4), while achieving the same VoI. This shows that guidelines can benefit from VoI and simulation to obtain cost-effective solutions that overcome the shortcomings of relying only on past operational experience.

The paper is organized as follows. Section 2 introduces the VoI concept and presents the framework that uses VoI and simulation for optimal positioning of sensors. Section 3 illustrates the case study that consists in the positioning of sensors on a SG; the solution for sensors positioning obtained following the guideline in UNI 11096 is benchmarked with that derived from the proposed VoI-based approach. Section 4 concludes the paper with some remarks.

\section{Value of Information}

Value of Information ( $\mathrm{VoI}$ ) is a mathematical concept used in Bayesian statistical decision theory to quantify (in monetary terms) the gain that one could obtain by updating prior available information with new one, before adopting it (21). Indeed, the process of acquiring information (here specifically consisting in measurements from sensors) may not always be justified because of the high cost, and one finds this out 
only after (the information is acquired by the measurements taken) (22,23). In other words, VoI predicts (by simulation) the economic benefit of collecting measurements in specific sensors locations, by accounting (in addition to the cost of the sensor and measurement acquisition chain) for the costs that the decision-maker might incur when adopting mitigative actions to counteract SSC degradation (or its failure, in case no action is taken) based on the collected measurements. This provides a powerful tool for comparing different locations of measurement before physically placing the sensors on the SSCs (i.e., a location that has larger VoI value is more beneficial) and makes it possible to find the most beneficial set by solving an optimization problem that finds the observation that has the largest VoI.

The mathematical framework for quantifying the VoI is recalled in the following: In general terms, a spatial domain $\Omega_{x}$ can be defined over the SSC of interest and indicated by its spatial coordinates. For example, each location on a 2-dimensional $\Omega_{x}$ can be indicated as $\bar{x}=\left\{x^{1}, x^{2}\right\}$. For practicality, the spatial domain $\Omega_{x}$ is discretized into a finite set of $i$ locations $\bar{X}=\left\{\bar{x}_{1}, \bar{x}_{2}, \ldots, \bar{x}_{i}\right\}$. A spatial model $F=f(\bar{x})$ can be introduced on the spatial domain $\Omega_{x}$ to measure a certain property of the SSC at each location $\bar{x}$ (e.g., for a pressurized vessel, a random field $f(\bar{x})$ of stress, at each location $\bar{x}$ ). If multiple random fields $\bar{f}(\bar{x})$ (e.g., stress, temperature, thickness and so on) insist on the SSC, $F=\bar{f}(\bar{x})$ can be defined as a multivariate random field, possibly with dependencies (24).

The spatial model can be described based on design values and operational experience (i.e., prior knowledge), eventually assigning prior distributions $\bar{p}_{F}$ of values to the relevant variables (e.g., the distribution of the internal stress in a pressurized vessel).

Similar to the spatial domain $\Omega_{x}$, the measurements spatial domain $\Omega_{y}$ can be defined at locations $\bar{x}=\left\{x^{1}\right.$, $\left.x^{2}\right\}$ where the measurements can be taken (i.e., $\Omega_{y} \subseteq \Omega_{x}$ ). The measurement set $\bar{y}(\bar{x})$ is the collection of measurements at the generic location $\bar{x}$ (e.g., measurements of realizations of the multivariate random field $\bar{f}(\bar{x}))$. Then, $\overline{\mathrm{Y}}$ is defined as the set of measurements $\bar{y}(\bar{x})$ at the selected locations $\bar{x}$ :

$$
\bar{y}(\bar{x})=\mathrm{R} \bar{f}(\bar{x})+\bar{\varepsilon}
$$

where $\mathrm{R}$ is a row matrix indicating which locations in $\boldsymbol{\Omega}_{x}$ are observed (i.e., $\mathrm{R}$ is a row matrix with zero 
value for the non-observed locations of $\bar{X}$ and 1 for the observed locations), and $\bar{\varepsilon}$ is a vector of random noise measurements, usually assumed to be distributed like a Gaussian with zero mean and covariance matrix $\Sigma_{\varepsilon}(24)$. When new information become available (new measurements are recorded), the distribution of the model $\bar{f}(\bar{x})$ can be updated. Specifically, Bayesian inference allows updating the prior distributions $\bar{p}_{F}$ to obtain the posterior distribution $\bar{p}_{F \mid \bar{y}}$.

Prior and posterior distributions, $\bar{p}_{F}$ and $\bar{p}_{F \mid \bar{y}}$ can be used by a decision-maker to decide an action from a set of possible actions $\bar{a}$. In particular, the SSCs prior probability of failure $\bar{P}_{F}(\bar{x})$ and posterior probability of failure $\bar{P}_{F \mid \bar{y}}(\bar{x})$ that can be inferred from $\bar{p}_{F}$ and $\bar{p}_{F \mid \bar{y}}$, respectively, can support, in a risk-informed perspective, a decision-maker choice of maintenance by balancing risk and actions costs, on the basis of the most informative set that can be collected.

To this aim, a loss function $L(\bar{f}(\bar{x}), \bar{a})$ can be introduced as negative utility (i.e., loss) that may come from taking a decision. For example, in case a decision of action is taken based only on the prior knowledge $\bar{p}_{F}$ without relying on additional measurements $(\varnothing)$, the prior expected loss $\mathbb{E L}(\varnothing)$ can be minimized to find the optimal action:

$$
\mathbb{E} L(\varnothing)=\min \left\{\mathbb{E}_{F} L(\bar{f}(\bar{x}), \bar{a})\right\}
$$

On the other hand, if $\bar{y}(\bar{x})$ is available, the decision can be taken a posteriori of collecting the information $\bar{y}(\bar{x})$, i.e., with respect to $\bar{p}_{F \mid \bar{y}}$, and minimizing the posterior expected loss $\mathbb{E} L(\bar{y}(\bar{x}))$ :

$$
\mathbb{E} L(\bar{y}(\bar{x}))=\mathbb{E}_{Y} \min \left\{\mathbb{E}_{F \mid \bar{y}} L(\bar{f}(\bar{x}), \bar{a})\right\}
$$

For example, based on $\bar{p}_{F}$ (e.g. the prior knowledge on the field of stress on a plate with growing cracks, providing a failure probability estimate $\bar{P}_{F}(\bar{x})$ due to the load applied) a repair decision might be taken that differs from the one that would have been taken if the posterior probability of failure $\bar{P}_{F \mid \bar{y}}(\bar{x})$ would have been considered, if $\bar{P}_{F \mid \bar{y}}(\bar{x})$ were updated with the new measurements $\bar{y}(\bar{x})$ that have become available.

For the pressurized vessel, $\bar{p}_{F \mid \bar{y}}$ reflects the updated distribution of the stress, conditioned on the collected information $\bar{y}(\bar{x})$ : within the here proposed simulation-based approach, the measurement corresponds to a 
random realizations in a specific location $i$. To account for this stochasticity, we assume that in each location, we simulate the collection of $K$ alternative measurements, and $\bar{p}_{F}$ and $\bar{p}_{F \mid \bar{y}}$ are collected accordingly with their consequent losses as in Equations (2) and (3). $K$ different stochastic realizations of $\bar{y}\left(\bar{x}_{i}\right)$ represent $K$ different posterior expected losses $\left(\mathbb{E} L\left(\bar{y}\left(\bar{x}_{i}\right)\right)_{k}\right.$ which are averaged to quantify the posterior expected loss conditioned on the measurement at a specific location $i$ as:

$$
\mathbb{E} L\left(\bar{y}\left(\bar{x}_{i}\right)\right)=\frac{\sum_{k=1}^{K}\left(\mathbb{E} L\left(\bar{y}\left(\bar{x}_{i}\right)\right)_{k}\right.}{K}
$$

The difference between $\mathbb{E L}(\varnothing)$ and $\mathbb{E} L(\bar{y}(\bar{x}))$ quantifies the benefit of taking decisions informed by the new information $\bar{y}(\bar{x})$, and is, thus, the VoI:

$$
\operatorname{VoI}(\bar{y}(\bar{x}))=\mathbb{E L}(\varnothing)-\mathbb{E L}(\bar{y}(\bar{x}))
$$

\subsection{VoI-based sensor positioning}

As mentioned before, decisions are made optimal by the informativeness of $\bar{y}(\bar{x})$. The cost-effectiveness of $\bar{y}(\bar{x})$ holds when its cost $\mathrm{C}(\bar{y}(\bar{x}))$ is less than (or equal) to the $\operatorname{VoI}$ gained (i.e., $\operatorname{VoI}(\bar{y}(\bar{x})) \geq \mathrm{C}(\bar{y}(\bar{x}))$ ), and the utility $\mathrm{M}(\bar{y}(\bar{x})) \geq 0$ :

$$
\mathrm{M}(\bar{y}(\bar{x}))=\operatorname{VoI}(\bar{y}(\bar{x}))-\mathrm{C}(\bar{y}(\bar{x}))
$$

The optimal set of measurement $\bar{y}\left(\bar{x}^{*}\right)$, i.e., the set which maximizes the utility $\mathrm{M}(\bar{y}(\bar{x}))$, is determined by the optimal number of sensors $n$ and their positioning. In principle, the optimal positioning of sensors can be found by simulating every possible set of measurement location on $\Omega_{y}$ by a combinatorial and computationally impractical way of solving Equation (7):

$$
\bar{y}^{*}(\bar{x})=\operatorname{argmax}_{\bar{Y} \subseteq \Omega_{Y}}(M(\bar{Y}))
$$

Alternatively, optimization approaches can be used: in greedy optimization approach, firstly the optimal positioning for a single sensor is searched by simulation (i.e., $n=1$ ) and, then, based on this result, the next sensor is positioned until, iteratively, the maximum desired $\mathrm{M}(\bar{Y})$ is reached by updating the $n_{\text {th }} \bar{p}_{F}$ from the $(n-1)_{t h} \bar{p}_{F \mid \bar{y}}$ to take into account the availability of new measurements taken at the selected location $(25,26)$.

\subsection{VoI-based sensor positioning for Gaussian fields}


A particular case of the sensor positioning procedure described in Section 2.1 is the sensor positioning on Gaussian fields, which means that $\bar{f}(\bar{x})$ is normally distributed on $\Omega_{x}(27)$, with a mean function value $m(\bar{x})$, a standard deviation $\sigma(\bar{x})$, and covariance $k\left(\bar{x}, \bar{x}^{\prime}\right)$ with correlation $\rho(\bar{x}, \bar{x})$ (i.e., $k\left(\bar{x}, \bar{x}^{\prime}\right)=\sigma(\bar{x}) \sigma\left(\bar{x}^{\prime}\right) \rho\left(\bar{x}, \bar{x}^{\prime}\right)$ ) between locations $\bar{x}$ and $\bar{x}^{\prime}$. This means, also, that when a measurement is taken at a given location $\left(x_{1}, x_{2}\right)$, any other measurement at any other location $\left(x_{1}{ }^{\prime}, x_{2}{ }^{\prime}\right)$ is correlated with $\left(x_{1}, x_{2}\right)$ according to the exponential correlation function (28):

$$
\rho\left(x_{1}, x_{2}, x_{1}^{\prime}, x_{2}^{\prime}\right)=\exp \left(-\sqrt{\frac{\left(\left(x_{1}-x_{1}^{\prime}\right)^{2}+\left(x_{2}-x_{2}^{\prime}\right)^{2}\right)}{\lambda^{2}}}\right)
$$

where $\lambda$ is called scale parameter (in the case study of Section $3, \lambda$ is taken equal to $100 \mathrm{~mm}$ ).

In various cases, spatially distributed systems can be assumed to be Gaussian $(29,28)$ and, Bayesian inference for the sensors positioning can exploit the property of conjugate priors (30), as discussed hereafter.

Specifically:

- the multivariate field $\bar{f}(\bar{x})$ can be described by the mean vector $\bar{\mu}_{F}=\bar{m}(\bar{x})$ containing multivariate mean values $m(\bar{x})$ of different SSC properties and a covariance matrix $\Sigma_{\mathrm{F}}=k\left(\bar{x}, \bar{x}^{\prime}\right)$, with a prior distribution $\overline{p_{F}}$ :

$$
\bar{f}(\bar{x}) \sim \bar{p}_{F}=\mathbb{N}\left(\bar{\mu}_{F}, \Sigma_{F}\right)
$$

- the measurement set $\bar{y}(\bar{x})$ of Equation (1) is modeled as an independent, identically distributed, zero mean Gaussian distribution covariance matrix $\Sigma_{\varepsilon}$ can be described as distributed by a multivariate normal distribution with $\bar{\mu}_{\bar{Y}}=\mathrm{R} \bar{\mu}_{F}, \Sigma_{\bar{Y}}=\mathrm{R} \Sigma_{\mathrm{F}} \mathrm{R}^{\mathrm{T}}+\Sigma_{\varepsilon}$ (Equation (10)):

$$
\bar{y}(\bar{x}) \sim \bar{p}_{F \mid \bar{y}}=\mathbb{N}\left(\bar{\mu}_{\bar{Y}}, \Sigma_{\bar{Y}}\right)
$$

The conjugate posterior of $\bar{f}(\bar{x})$ is, thus, a Gaussian distribution (31):

$$
\bar{f} \mid \bar{y}(\bar{x}) \sim \mathbb{N}\left(\bar{\mu}_{F \mid \bar{y}}, \Sigma_{F \mid \bar{Y}}\right)
$$

with:

$$
\bar{\mu}_{F \mid \bar{y}}=\bar{\mu}_{F}+\Sigma_{F} \mathrm{R}^{\mathrm{T}} \Sigma_{\bar{Y}}^{-1}\left(\bar{y}(\bar{x})-\bar{\mu}_{\bar{Y}}\right)
$$


and

$$
\Sigma_{F \mid \bar{Y}}=\Sigma_{F}-\Sigma_{F} \mathrm{R}^{\mathrm{T}} \Sigma_{\bar{Y}}^{-1} \mathrm{R} \Sigma_{F}
$$

being the posterior mean and covariance, respectively.

\section{Application of the VoI-based Approach for Optimal Sensor Positioning on a SG Undergoing Creep}

The application of the VoI-based approach for sensor positioning described in Section 2.1. is here shown with respect to the optimization of sensors positioning on a manifold of the SG of a Prototype Fast Breeder Reactor (PFBR) (See Figure 1), whose thickness can be measured by Ultrasonic Thickness Testing (UTS).

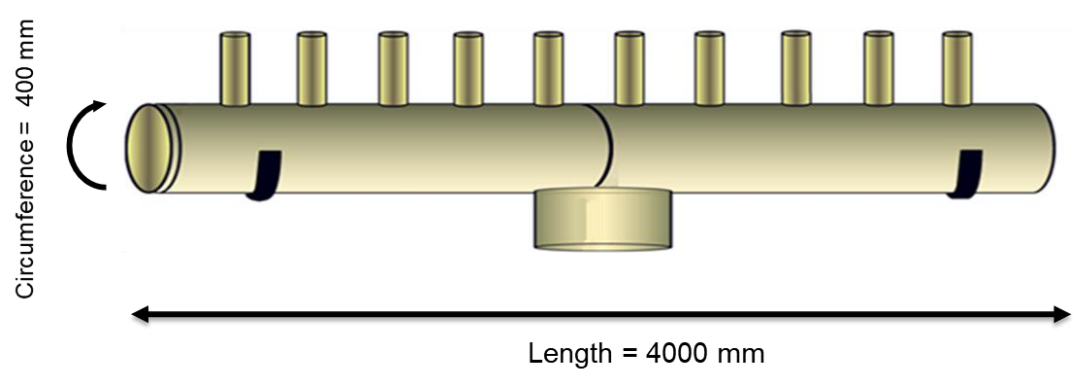

Figure 1:The manifold of a SG of a PFBR used as test case for the VoI-based approach for sensor positioning

Under the assumed operating conditions listed in Table 1, the manifold may suffer of creep due to the large design pressure and temperature, and long exposure time that may lead to failure.

Table 1: Operating conditions of the SG

\begin{tabular}{|c|c|}
\hline Design pressure & 189 barg \\
\hline Design temperature & $\begin{array}{c}778^{\circ} \mathrm{K}=505^{\circ} \mathrm{C} \quad \text { inlet } \\
723^{\circ} \mathrm{K}=450{ }^{\circ} \mathrm{C} \quad \text { outlet }\end{array}$ \\
\hline Material & $\begin{array}{c}\text { 9Cr-1Mo-V-Nb (Plate) } \\
\text { ASME SA-387/SA-387M Grade } 91\end{array}$ \\
\hline $\begin{array}{c}\text { Percentage of life } \\
\text { spent }\end{array}$ & $35 \%$ \\
\hline Operating hours & $100,000 \mathrm{~h}$ \\
\hline Tensile strength & $475 \mathrm{MPa}$ \\
\hline Thickness & $20 \mathrm{~mm}$ \\
\hline
\end{tabular}

Failure would occur at location $\bar{x}$ if the thickness (i.e., the Gaussian strength random field $f_{s}(\bar{x})$ ) is smaller than a threshold thickness, here assumed equal to a constant value of $16.9 \mathrm{~mm}$ on the whole $\Omega_{x}$ (i.e., the 
failure threshold field $f_{t}(\bar{x})$ calculated in (32) in line with (33) using the NIMS creep database (34), that contains creep data collected in experiments related to pressurized equipment of NPP of the same material and operating under the same conditions of the manifold considered in our case).

Since $f_{s}(\bar{x})$ is a Gaussian field and $f_{t}(\bar{x})$ is a constant, the limit state function $g(\bar{x})=f_{s}(\bar{x})-f_{t}(\bar{x})$ is a Gaussian $g(\bar{x}) \sim \mathrm{N}\left(\mu_{\mathrm{g}}(\bar{x}), \sigma_{\mathrm{g}}(\bar{x})\right)$, which implies a probability of manifold failure $\bar{P}(\bar{x})$ (i.e. the probability that $\mathrm{g}(\bar{x})<0)$ equal to:

$$
\bar{P}(\bar{x})=\Phi(-\beta(\bar{x}))
$$

where $\Phi($.$) is the standard normal cumulative distribution function, and \beta$ is the reliability index equal to:

$$
\beta(\bar{x})=\frac{\mu_{g}(\bar{x})}{\sigma_{g}(\bar{x})}
$$

The technical procedure in the Italian guideline ISPESL n. 48/2003 (33) and, specifically, the norm (UNI 11096, 2012) (4) is used as benchmark for the sensors positioning. In line with (4), 32 thickness gauges are placed in the locations $\left(^{*}\right)$ of Figure 2, among 160 locations available. Notice that holes of subchannels within the manifold are neglected in line with (4).

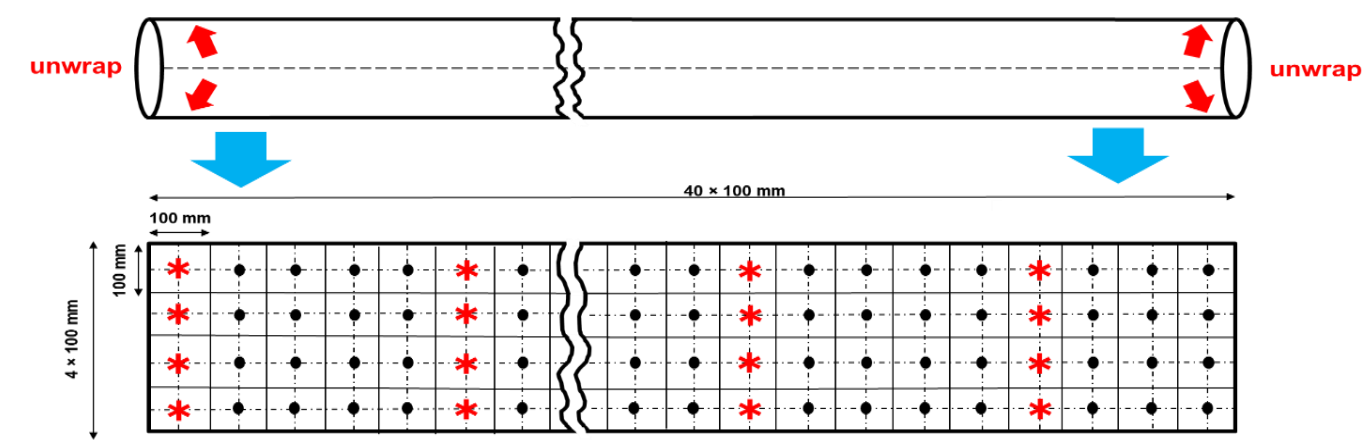

Figure 2: Schematic view of the unwrapped manifold with sensor locations $(\bar{x})$, in line with (UNI 11096, 2012).

This benchmark positioning is, however, independent from the actual $f_{s}(\bar{x})$ that may vary, due to the manifold production process. The following common cases are considered (see Figure 3):

1. Circumferential welding of two extruded manifolds.

2. Longitudinal welding of a rectangular plate.

3. Circumferential welding of two manifolds resulting from a longitudinal welding of two rectangular plates. 


\section{Manifold extrusion.}

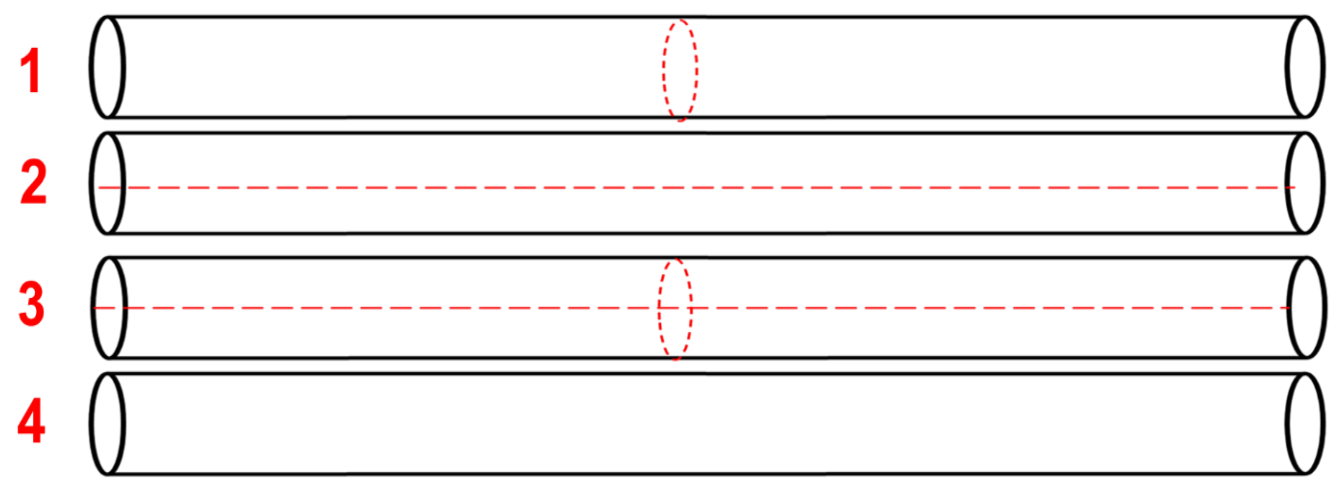

Figure 3: schematic view of the four case studies.

In all these cases, the $f_{s}(\bar{x})$ is modeled with a Gaussian model $\bar{p}_{F}(\bar{x})=\mathbb{N} \sim(20 \mathrm{~mm}, 1 \mathrm{~mm})$ except for the welding and Heat Affected Zone (HAZ), where $\bar{p}_{F}(\bar{x})$ of $f_{s}(\bar{x})$ is $\mathbb{N} \sim(20 \mathrm{~mm}, 2 \mathrm{~mm})$. Figure 4 shows the standard deviation of $f_{s}(\bar{x})$ for all the cases (the lighter the color, the larger the standard deviation).

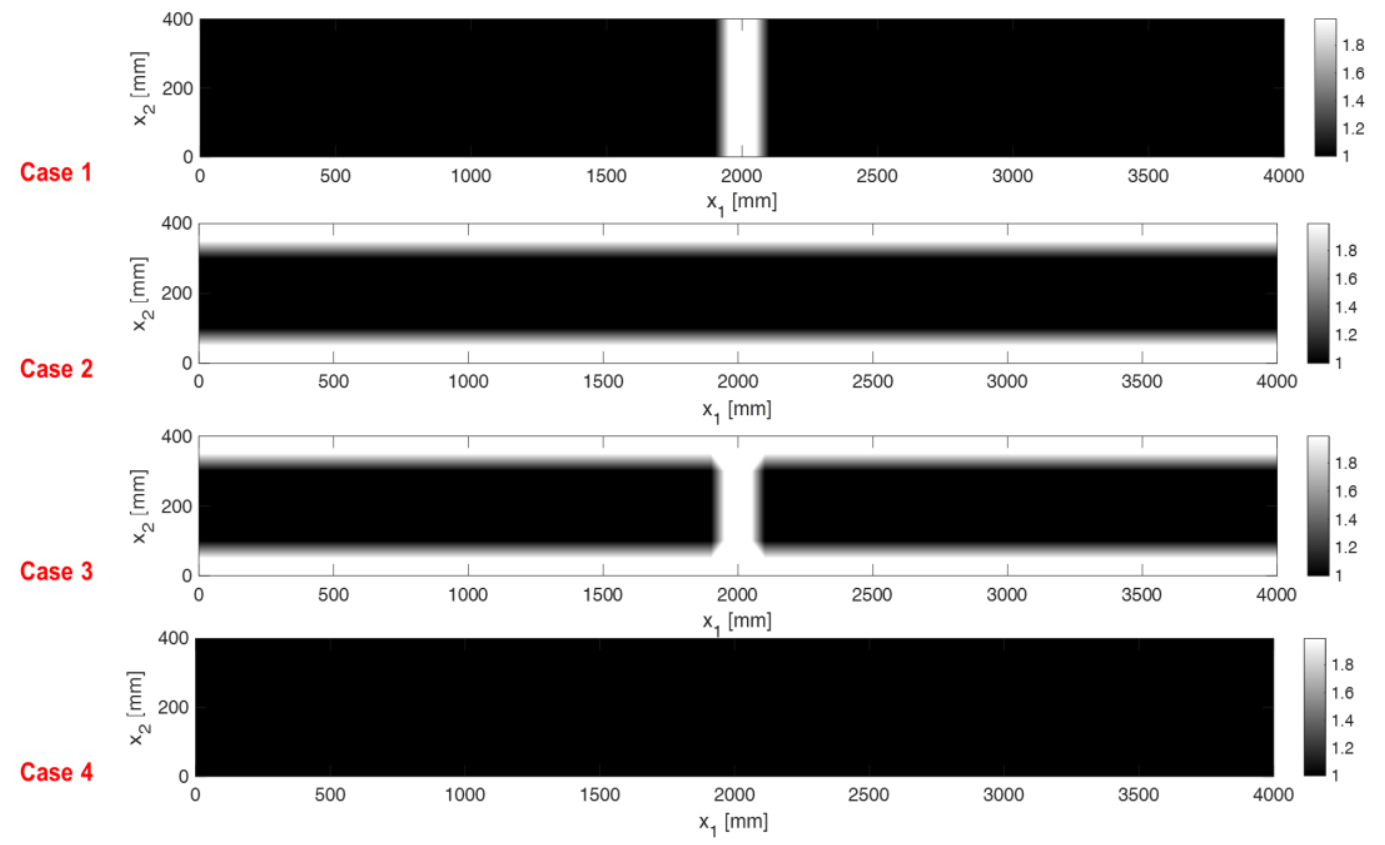

Figure 4: Standard deviation of $f_{s}(\bar{x})$ for the four case studies

Knowing that $f_{t}(\bar{x})$ is equal to $16.9 \mathrm{~mm}$, the prior probability of failure $\bar{P}_{F}(\bar{x})$ can be calculated at any location $\bar{x}$ of the manifold, as plotted in Figure 5 (the warmer the color, the larger the probability of failure). 

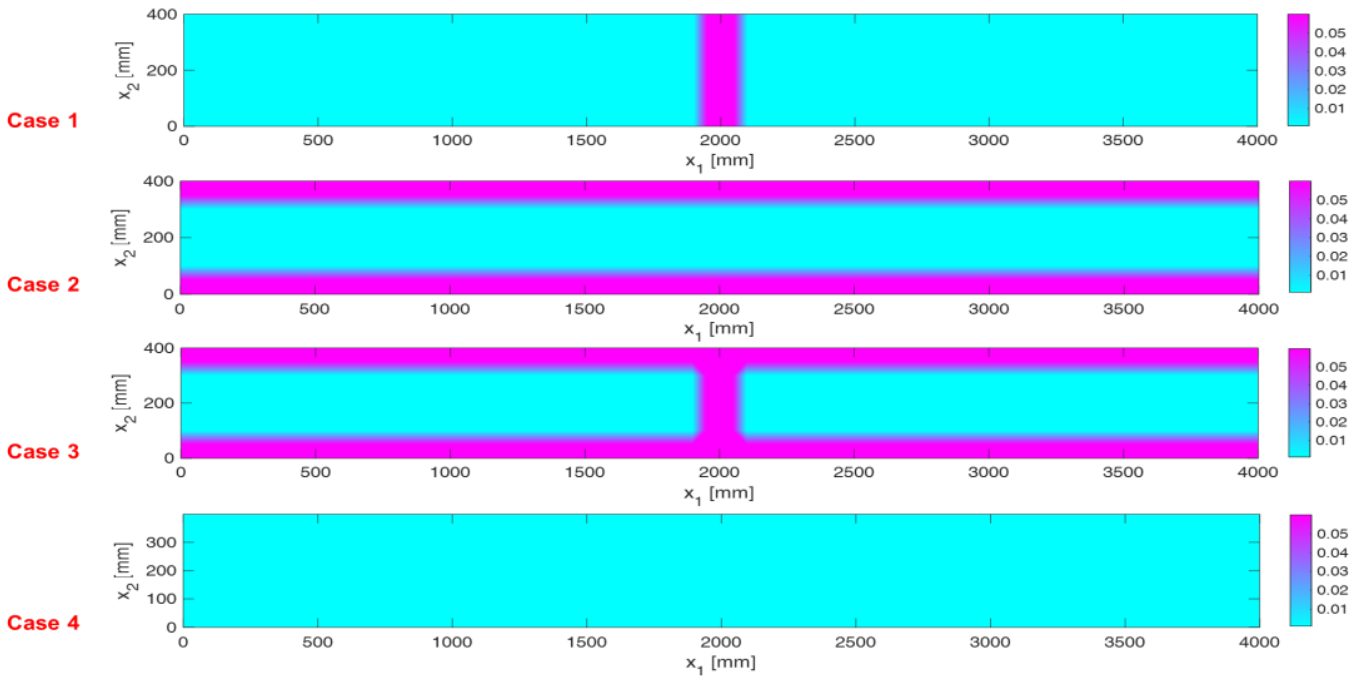

Figure 5: Prior probability of failure $\bar{P}_{F}(\bar{x})$ for the four case studies

At this point, it can be decided to: 1 . do nothing $(a=0)$ with zero cost, or 2. mitigate degradation $(a=1)$ (for, example, for mitigating creep, one can either reduce the operational stress (i.e., lowering pressure, temperature, ...), or sleeve the risky area, or perform a weld repair (35), or any combination of these with cost $C_{m}$ ). Depending on the true state $(\bar{s})$ of the manifold, unknown to the decision maker, (i.e., too much degraded ( $(s=0)$ that would entail failure cost of $C_{f}$, or operational $(s=1)$ ), one sets the loss function value:

$$
L(\bar{f}(\bar{x}), \bar{a})=\left\{\begin{array}{lr}
0 & \text { if } s=1 \text { and } a=0 \\
C_{f}=200 K € & \text { if } s=0 \text { and } a=0 \\
C_{m}=5 K € & \text { if } a=1
\end{array}\right\}
$$

In other words, if no mitigation action is performed $(a=0)$ and the true state is operational $(s=1)$, then the decision comes with zero cost; otherwise, if the actual state is too degraded $(s=0)$, then, a wrong decision comes with cost $C_{f}$. It is assumed that, regardless of the true state of the manifold, if a failure mitigation action is undertaken, the payoff is the $\operatorname{cost} C_{m}$.

For the proposed application, the prior expected loss $\mathbb{E} L(\emptyset)=\sum_{i=1}^{160} \mathbb{E} L_{i}(\varnothing)$ is quantified by simultaneously accounting for all the 160 prior expected losses, where, for the $i$-th location, the prior expected loss is:

$$
\mathbb{E} L_{i}(\varnothing)=\min \left\{\mathbb{E}_{F} L_{i}\left(\bar{f}\left(\bar{x}_{i}\right), \bar{a}_{i}\right)\right\}=\min \left\{\mathbb{E}_{F} L_{i}\left(\bar{f}\left(\bar{x}_{i}\right), 0\right), \mathbb{E}_{F} L_{i}\left(\bar{f}\left(\bar{x}_{i}\right), 1\right)\right\}=\min \left\{C_{f} \times P_{F}\left(\bar{x}_{i}\right), C_{m}\right\}
$$

being $\mathbb{E}_{F} L_{i}\left(\bar{f}\left(\bar{x}_{i}\right), 0\right)$ the failure cost $C_{f}=200 \mathrm{~K} €$ weighted by the probability of failure $P_{F}\left(\bar{x}_{i}\right)$ (if no action 
is taken, $a=0$ ), and $\mathbb{E}_{F} L_{i}\left(\bar{f}\left(\bar{x}_{i}\right), 1\right)$ corresponds to the cost $C_{m}=5 K €$ (if maintenance action is taken, $a=1$ ). Also the posterior expected loss $\mathbb{E} L(\bar{y}(\bar{x}))=\sum_{i=1}^{160} \mathbb{E} L_{i}(\bar{y}(\bar{x}))$ is quantified by simultaneously accounting for all the 160 posterior expected losses where, for the $i$-th location, the posterior expected loss conditional on the measurement set $\bar{y}(\bar{x})$ is:

$$
\mathbb{E} L_{i}(\bar{y}(\bar{x}))=\min \left\{C_{f} \times P_{F \mid \bar{y}}\left(\bar{x}_{i}\right), C_{m}\right\}
$$

being $P_{F \mid \bar{y}}\left(\bar{x}_{i}\right)$ the posterior probability of failure of location $\bar{x}_{i}$.

It is worth mentioning that $\mathbb{E} L(\varnothing)$ and $\mathbb{E} L(\bar{y}(\bar{x}))$ have been calculated as the sum of all the prior and posterior losses, respectively, of all the 160 locations, because we conservatively assume that the manifold integrity is lost when at least one measurement location is overlooked ignoring the necessary mitigative action to counteract manifold failure.

In Figure 6 , the $\bar{P}_{F}$, the prior $\bar{a}$ and the prior expected loss of each $i_{\text {th }}$ location $\mathbb{E} L_{i}$ for the case 1 (circumferential welding of two extruded manifolds) are plotted resulting in a total prior expected loss $\mathbb{E} L(\varnothing)=\sum_{i=1}^{160} \mathbb{E} L_{\mathrm{i}}(\varnothing)$, which can be interpreted as the utility value of taking decisions $\bar{a}$ on the prior belief $\bar{p}_{F}$, when due account is given to all the 160 prior expected loss values $\mathbb{E} L_{i}$ of each $i_{\text {th }}$ discretized locations $\bar{x}$.
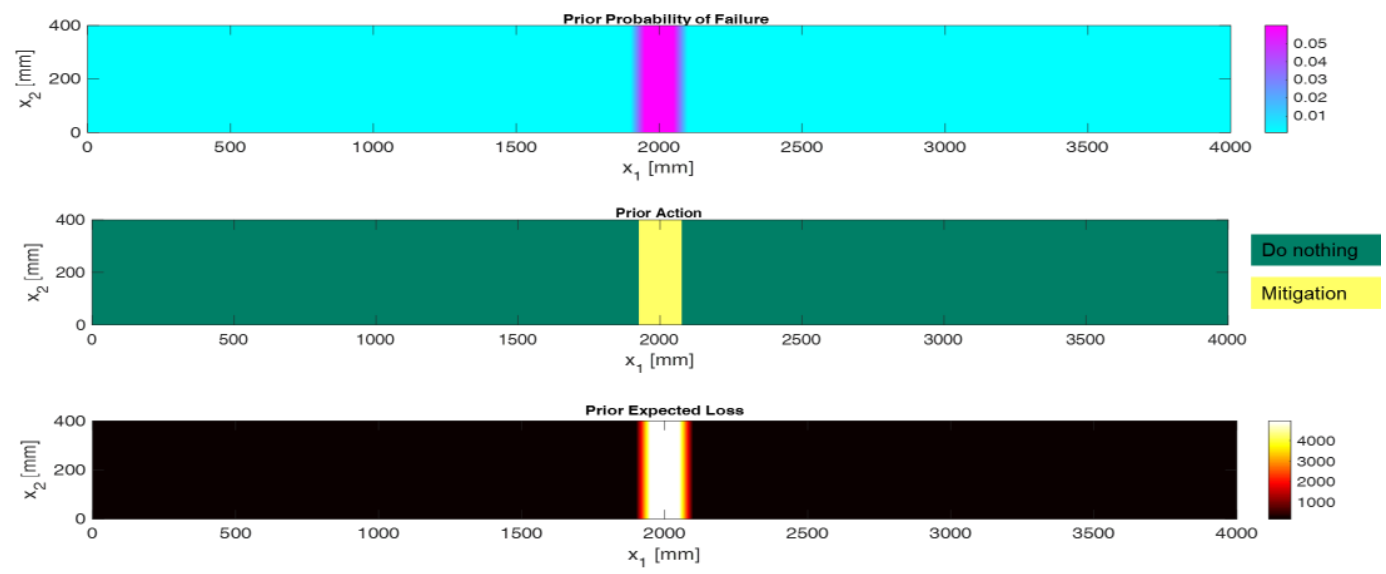

Figure 6: Prior probability, prior actions and the prior expected loss for the case study 1 
When a new measurement $\bar{y}(\bar{x})$ is taken, it reduces the uncertainty of $f(\bar{x})$. In each realization, $\bar{p}_{F}$ is to be updated in $\bar{p}_{F \mid \bar{y}}$ to calculate the posterior probability of failure $\bar{P}_{F \mid \bar{y}}$, the posterior actions $(\bar{a})$ and the posterior expected loss $\mathbb{E} L_{i}(\bar{y}(\bar{x}))$ for each $i_{\text {th }}$ location (see Figure 7, where the posterior expected loss field is plotted conditioned on a specific observation at location $\bar{x}_{o}$ shown by the circle). Finally, $\operatorname{VoI}(\bar{y}(\bar{x}))$ is calculated using Equation (5) and $\mathbb{E} L(\bar{y}(\bar{x}))=\sum_{i=1}^{160} E L_{i}(\bar{y}(\bar{x}))$.

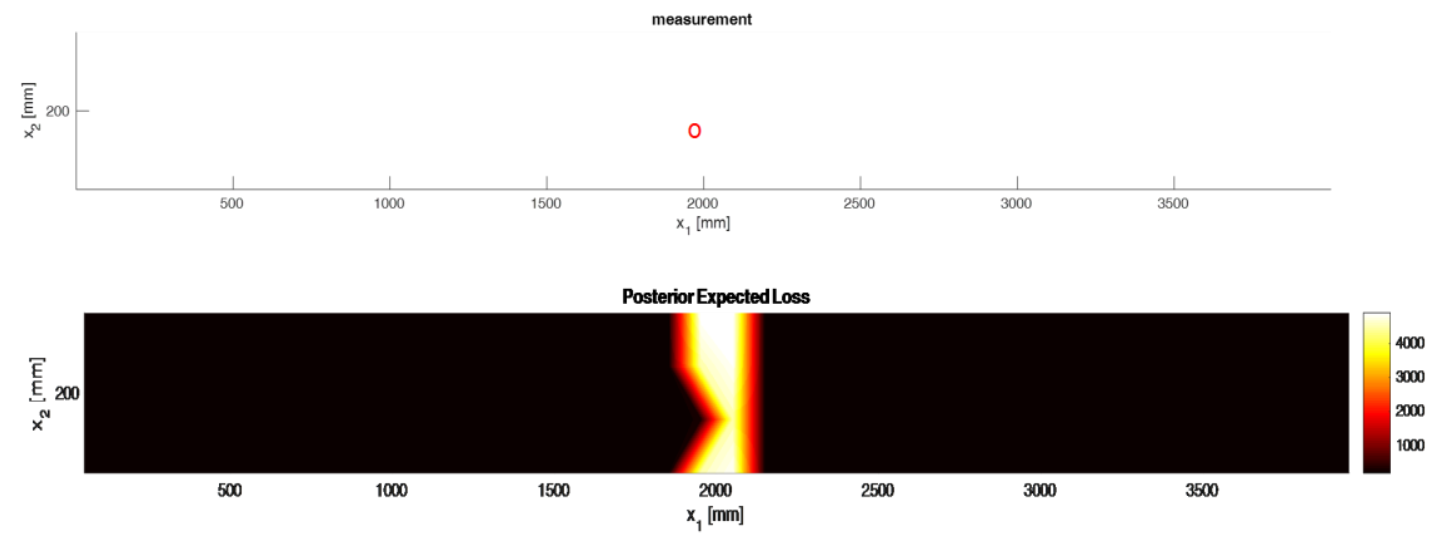

Figure 7: The plot of the posterior expected loss field, conditioned on a specific observation at location $\bar{x}_{0}$.

In this case study, $V_{0 I} I_{U N}$ is the standard value to be reached by the inspection scheme assuming that the inspection costs are neglected (i.e., $\mathrm{C}(\bar{y}(\bar{x}))=0$ ). As a result, the largest VoI of the case study comes from the set of measurement that fills all the $i=160$ candidate locations with sensors. Instead, we are looking for a set that its benefits exceeds that of the standard value $V_{o I} I_{U N}$ to evaluate the proposed simulation-based methodology's capabilities and compare it with the experience-based standard in recommending better sensors positioning strategy.

The optimal $n$ sensors locations $\bar{y}\left(\bar{x}^{*}\right)$ can be found with the greedy optimization method whose flowchart, tailored on the specific case study, is given in Figure 8. 


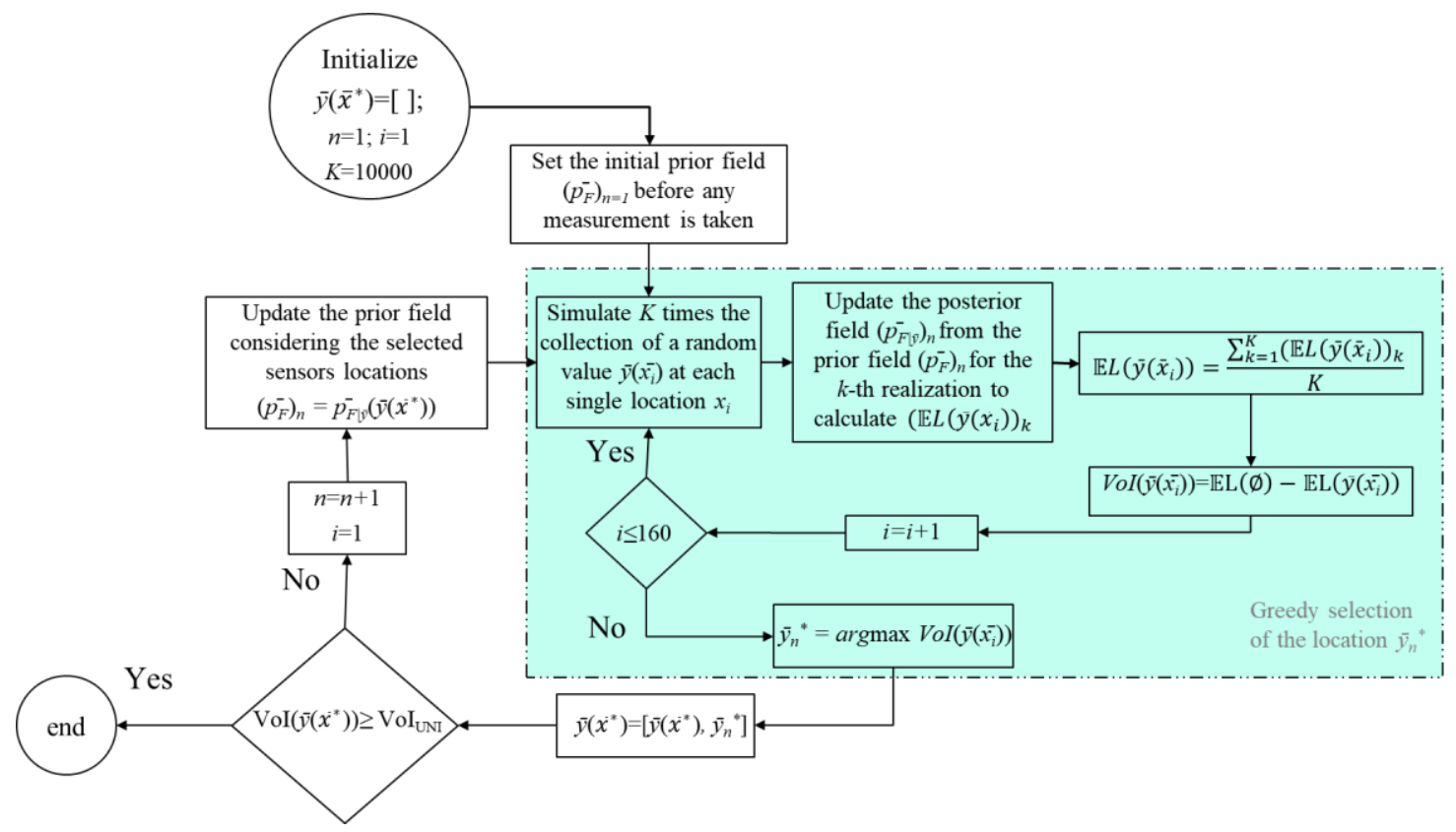

Figure 8: Flowchart for VoI-based greedy optimization

At each $n$-th iteration, we aim at positioning one sensor among the 160 candidate positions. The posterior field $\left(\bar{p}_{F \mid \bar{y}}\right)_{n}$ is calculated $K=10000$ times to simulate $K$ different stochastic realizations of $\bar{y}\left(\bar{x}_{i}\right)$. The posterior expected loss $\mathbb{E} L\left(\bar{y}\left(\bar{x}_{i}\right)\right)=\frac{\sum_{k=1}^{10000}\left(\mathbb{E} L\left(\bar{y}\left(\bar{x}_{i}\right)\right)_{k}\right.}{10000}$ (Equation (4)) is calculated and $\operatorname{VoI}\left(\bar{y}\left(\bar{x}_{i}\right)\right.$ is quantified; the sensor position $\bar{y}_{n}{ }^{*}$ that yields the largest $\operatorname{VoI}\left(\bar{y}\left(\bar{x}_{i}\right)\right)$ value among the 160 candidate positions is added to the optimal set $\bar{y}\left(\bar{x}^{*}\right)$ and the prior belief $\left(\bar{p}_{F}\right)_{n}$ of the next iteration becomes the posterior field, informed by $\bar{y}\left(\bar{x}^{*}\right)$ (i.e., $\left(\bar{p}_{F}\right)_{n}=\bar{p}_{F \mid \bar{y}}\left(\bar{y}\left(\bar{x}^{*}\right)\right.$ ). The search of the optimal location sets stops when the set $\bar{y}\left(\bar{x}^{*}\right)$ yields a VoI value greater than or equal to $V_{o I_{U N I}}$ that represents the positioning of 32 sensors in the 32 locations identified by $(*$ ) in Figure 2 (as supported by the technical procedure in the Italian guideline ISPESL n. 48/2003 (33) and, specifically, the norm (UNI 11096, 2012) (4), assuming that inspection costs are negligible with respect to $C_{m}$ and $C_{f}$ ).

Figure 9 shows the contour plots of $\mathrm{VoI}$ at the first iteration of greedy optimization, where the circle identifies the location where the max VoI would have been reached if the sensors were positioned there. 


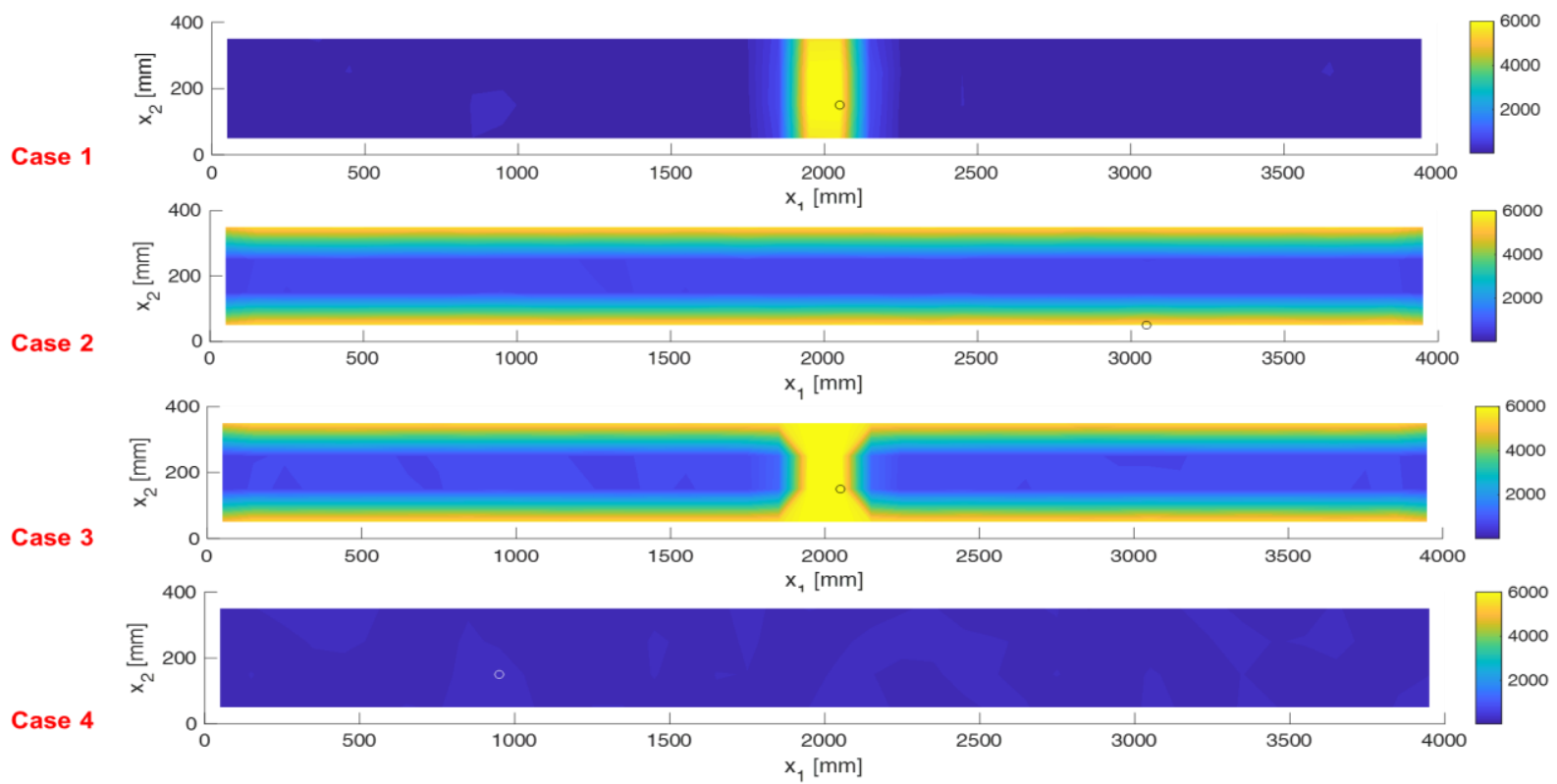

Figure 9: VoI contours for the four case studies at the first iteration $n=1$.

It can be seen that locations close to the HAZ (with larger uncertainty) also have larger VoI; a further proof comes from the case study 4 , for which all locations have the same $\bar{p}_{F}$ and, therefore, the VoI for any $i$-th location is almost identical with (all other locations (i.e., slight difference in VoI is due to the stochasticity of the random field characterization)).

Figure 10 shows the optimal sensors positions $\bar{y}\left(\bar{x}^{*}\right)$ for each of the case studies. The number of sensors $n$ required to exceed $V o I_{U N I}$ is $5,17,18,32$, respectively (where the numbers indicate the order of positions selected by the optimization strategy). 

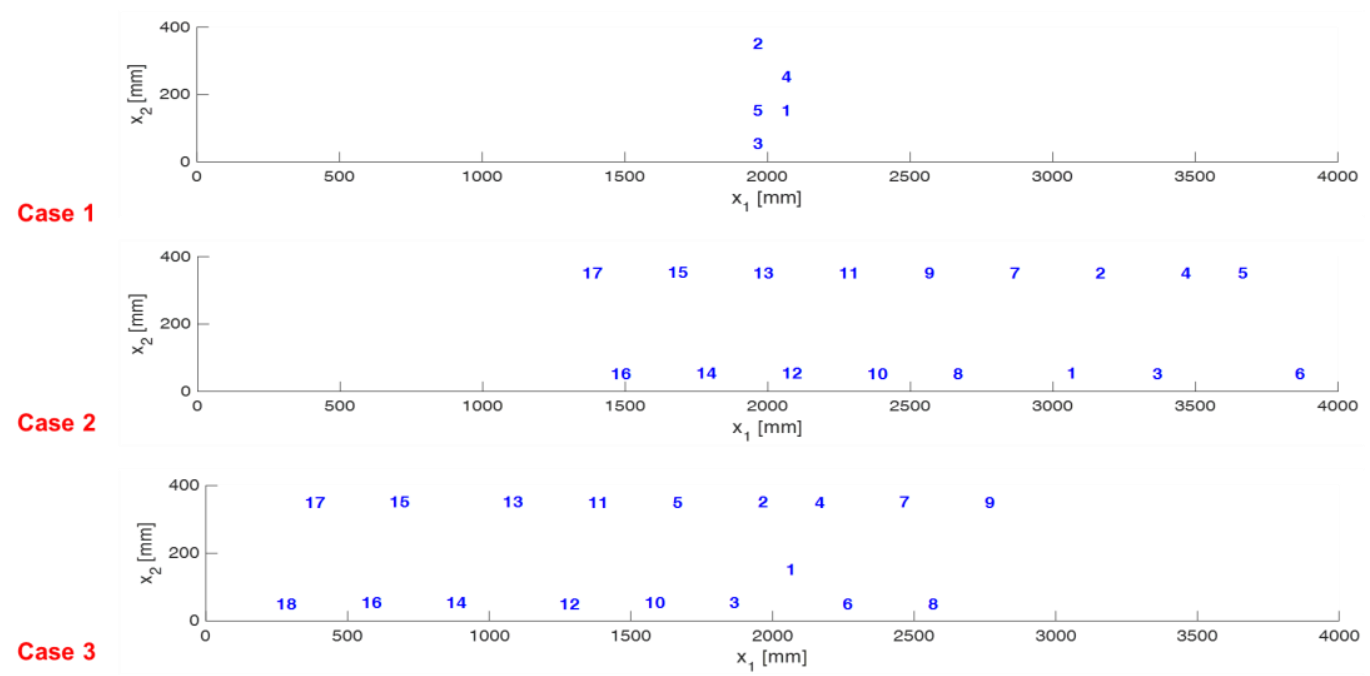

Case 3

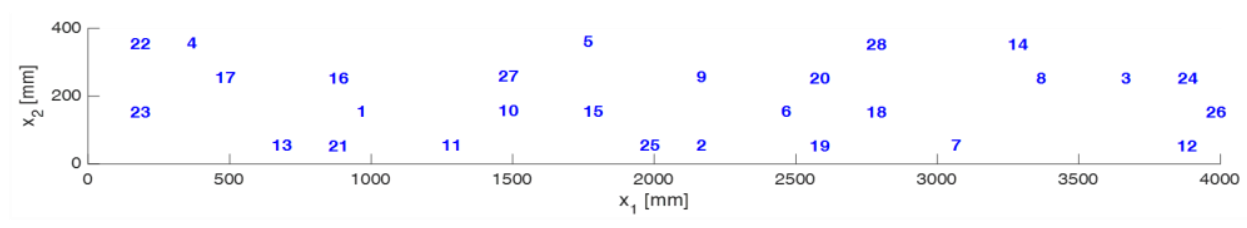

Figure 10: Sensor positioning using the greedy optimization algorithm for the four case studies

In all cases, $n<n_{\mathrm{UNI}}=32$ and the ratio $r=\frac{V o I}{n}$ is defined to show the VoI-per-sensor in each case study.

Similarly, $r_{U N I}=\frac{\text { VoI }_{\text {UNI }}}{n_{\text {UNI }}}$ shows the VoI-per-sensor obtained by positioning by the (4) guidelines with $n_{\mathrm{UNI}}=32$. Table 2 compares quantitatively the VoI, number of sensors $n$, and the VoI-per-sensor ratio $r$ in the different case studies, with their corresponding values of the (UNI 11096, 2012) sensors positioning. The VoI-per-sensor ratio $r$ of the case studies are compared with their associated $r_{U N I}$ values, with the coefficient of improvement $C_{I}$ showing how much the VoI-per-sensor ratio $r$ is improved using the suggested framework $\left(C_{I}=\frac{r}{r_{U N I}}\right)$.

Table 2: Comparison between the UNI 11096 and the proposed method for sensor positioning

\begin{tabular}{|c|c|c|c|c|c|c|c|}
\hline Case & VoI & $\boldsymbol{n}$ & $\boldsymbol{r}$ & $\boldsymbol{V o I}_{\text {UNI }}$ & $\boldsymbol{n}_{\mathrm{UNI}}$ & $\boldsymbol{r}_{\text {UNI }}$ & $C_{I}$ \\
\hline $\mathbf{1}$ & $2.6495 \mathrm{e}+04$ & 5 & 5299 & $2.6386 \mathrm{e}+04$ & 32 & 824.56 & 6.43 \\
\hline $\mathbf{2}$ & $9.9772 \mathrm{e}+04$ & 17 & 5868.94 & $9.4014 \mathrm{e}+04$ & 32 & 2937.94 & 2.00 \\
\hline
\end{tabular}




\begin{tabular}{|c|c|c|c|c|c|c|c|}
\hline $\mathbf{3}$ & $1.0727 \mathrm{e}+05$ & 18 & 5959.44 & $1.0420 \mathrm{e}+05$ & 32 & 3256.23 & 1.83 \\
\hline $\mathbf{4}$ & $6.1561 \mathrm{e}+03$ & 28 & 219.86 & $5.9847 \mathrm{e}+03$ & 32 & 187.02 & 1.18 \\
\hline
\end{tabular}

It can be seen that the VoI-based sensor positioning strategy in all cases gives a better VoI-per-sensor than following the normative recommendation of (4).

\subsection{Considerations on Geometrical Symmetry}

Due to the symmetry of the manifold geometry and thickness distribution, one might argue that positioning sensors in locations $n_{s}$ with the same uncertainty should not imply differences in VoI. To test this hypothesis, let us consider case study 1 , in which $n_{s}=8$ potential sensors locations fall in the HAZ area with large uncertainty (i.e., elements in the frame highlighted in Figure 11) and they may be equally selected as one of the optimal location of each greedy optimization iteration (i.e., $\bar{y}_{n}{ }^{*}$ ). These locations are sketched in Figure 11 (top).
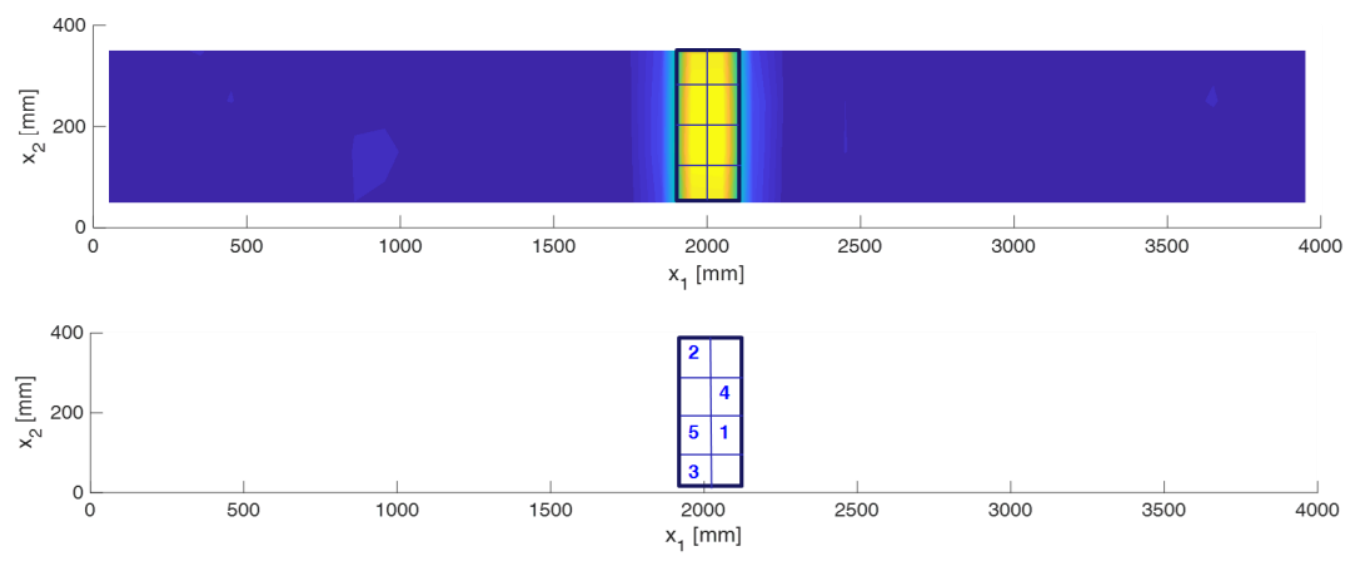

Figure 11: Sensor positioning for case study 1

As mentioned in Section 3, to exceed the $\operatorname{VoI}_{U N I}$ value of case study 1, a measurement set $\bar{y}\left(\bar{x}^{*}\right)$ comprised of $n=5$ sensors was needed (shown in Figure 11 (bottom)). However, if the sensor positioning approach is repeated 1000 times to find $\bar{y}\left(\bar{x}^{*}\right)$ and the probability that each one of these $n_{s}$ locations being selected as one of the $n$ optimal sensors of $\bar{y}\left(\bar{x}^{*}\right)$ is calculated, the results shown in Figure 12 are obtained, showing that the probability of each location being selected as one of the $n$ optimal sensors positionings is $62 \pm 5 \%$, which 
proves that the locations $n_{s}$ sharing the same uncertainty and geometrical properties have almost equal probability to be selected as one of the optimized locations for sensor positioning of the optimal set $\bar{y}\left(\bar{x}^{*}\right)$.

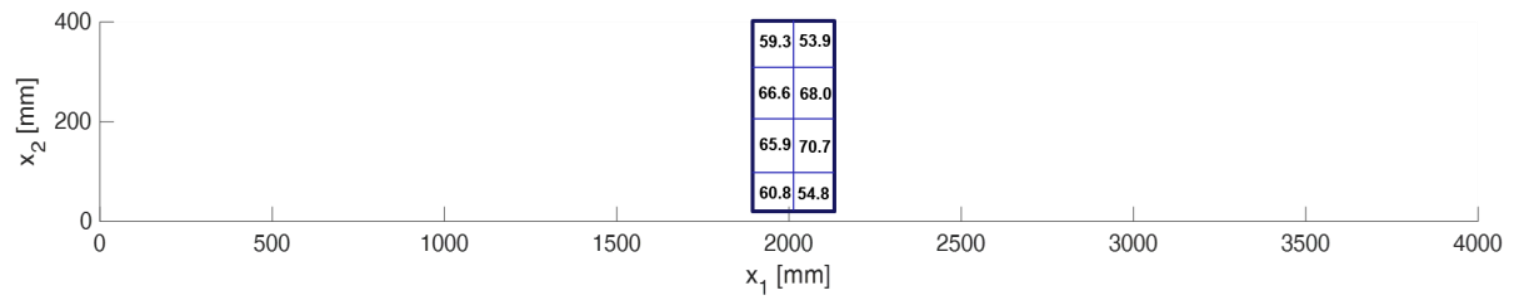

Figure 12: Probability of the sensors locations to be selected as one of the optimal positions (\%)

\section{Conclusions}

In this paper, a VoI-based, simulation-optimization framework has been presented for optimal sensors positioning. The framework has been applied to position the optimal sensors for monitoring the condition of a pressurized equipment degrading under creep. The main limitation of the proposed simulationoptimization framework is that it relies on a greedy optimization method which might encounter difficulties in some applications on a non-sub-modular metric like VoI (26). Despite this, in the application considered, the sensors positioning obtained using the proposed framework gives results that not only justify the positioning of the standard (4), but also require less sensors to reach the VoI that would be obtained by duly implementing the current guidelines/norms. Then, the proposed advanced computational framework can be used for supporting the development of guidelines based on past operational experience and could be particularly useful for those ones with limited past operational records.

\section{ACKNOWLEDGMENT}

The work is developed within the research project "SMART MAINTENANCE OF INDUSTRIAL PLANTS AND CIVIL STRUCTURES BY 4.0 MONITORING TECHNOLOGIES AND PROGNOSTIC APPROACHES - MAC4PRO ", sponsored by the call BRIC-2018 of the National Institute for Insurance 
against Accidents at Work - INAIL (CUP: J56C18002030001).

\section{REFERENCES}

1. Jelwan J, Chowdhury M, Pearce G. Creep life design criterion and its applications to pressure vessel codes. Materials Physics and Mechanics. 2011; 11(2): p. 157-182.

2. Stewart M. 2 - Piping standards, codes, and recommended practices. In Surface Production Operations: Volume 3: Facility Piping and Pipeline System.: Gulf Professional Publishing; 2016. p. 17-158.

3. Drummond MF. Introduction: the rationale for guidelines. Drug Information Journal. 1996; 30(2): p. 491-493.

4. UNI 11096. Non destructive testing - Structural integrity inspection of pressure equipment under creep condition - Inspection planning and execution, results evaluation and report. Rome:; 2012.

5. EN 13445. Unfired pressure vessels. ; 2018.

6. ASME. Boiler and Pressure Vessel Code. ; 2017.

7. Auerkari P, Brown TB, Holdsworth S, Hales R, Klenk A, Patel R, et al. Developments in assessing creep behaviour and creep life of components. Materials at High Temperatures 1. 2004; 21(1): p. 6164.

8. Delle Site C, Petris CD, Mennuti C. NDT tools for life assessment of high temperature pressure components. In ; 2006; Berlin, Germany: Proc. 9th European NDT (ECNDT) Conf.

9. Sposito G,WC, Cawley P, Nagy PB, Scruby C. A review of non-destructive techniques for the detection of creep damage in power plant steels. Ndt \& E International. 2010; 43(7): p. 555-567.

10. Baylac G, Kiesewetter N, Zeman J, Handtschoewercker A, McFarlane R, Corrado DS, et al. Creep Amendments in the European Standard EN 13445. In ; 2007: ASME 2007 Pressure Vessels and Piping Conference, pp.

11. Jaske CE, Topalis P, Loong WS, Sidek ASM. Risk Based Inspection Methodology for Components Subject to High-Temperature Creep. In ; 2017: ASME 2017 Pressure Vessels and Piping Conference.

12. Fong JT,N, Heckert A, Filliben JJ, Freiman. SW. A multi-scale failure-probability-based fatigue or creep rupture life model for estimating component co-reliability. International Journal of Pressure Vessels and Piping. 2019; 173 : p. 79-93.

13. Kumar A, Mathuriya S, Shilpi S. Detection of Creep Damage and Fatigue Failure in Thermal Power Plants and Pipelines by Non-Destructive Testing Techniques. A Review. International journal of engineering research and technology. 2014.

14. Tonti A, Site CD, Grisolia O, Franchi E. Italian Life-Assessment Procedure for Creep-Operated Pressure Equipment. In ; 2007; American Society of Mechanical Engineers.: ASME 2007 Pressure Vessels and Piping Conference. p. 535-540.

15. Faber MH. Risk and safety in civil engineering Zurich: Swiss Federal Institute of Technology ETH; 2007.

16. Daga R, Samal MK. Real-time monitoring of high temperature components. Procedia Engineering. 2013; 55: p. 421-427.

17. Rao GN. In-service inspection and structural health monitoring for safe and reliable operation of NPPs. Procedia Engineering. 2014; 86: p. 476-485. 
18. Noteboom JW, Hulshof HJM. On stream optical measurement to monitor creep strain. In ; 2017; London; United Kingdom: WCCM 2017 - 1st World Congress on Condition Monitoring.

19. Zio E. The future of risk assessment. Reliability Engineering \& System Safety. 2018; 177 : p. 176190.

20. DeGroot MH. Optimal statistical decisions Vol. 82: ohn Wiley \& Sons; 2005.

21. Raiffa H, Schlaifer R. Applied Statistical Decision Theory: Harvard University; 1961.

22. Straub D. Value of information analysis with structural reliability methods. Structural Safety. 2014; 49 : p. $75-85$.

23. Haladuick S, Dann. MR. Value of information-based decision analysis of the optimal next inspection type for deteriorating structural systems. Structure and Infrastructure Engineering. 2018; 14(9): p. 1283-1292.

24. Malings C, Pozzi M. Value of Information for Spatially Distributed Systems: application to sensor placement. Reliability Engineering \& System Safety. 2016a; 154: p. 219-233.

25. Malings C, Pozzi M. Submodularity issues in value-of-information-based sensor placement. Reliability Engineering \& System Safety. 2019; 183 : p. 93-103.

26. Hoseyni SM, Maio FD, E. Zio. VoI-Based Optimal Sensors Positioning and the Sub-Modularity Issue. In ; 2019; Rome, Italy: 4th International Conference on System Reliability and Safety (ICSRS), pp. 148-152.

27. Kocijan J. Modelling and control of dynamic systems using Gaussian process models: Springer International Publishing; 2016.

28. Malings C, Pozzi M. Value-of-information in spatio-temporal systems: Sensor placement and scheduling. Reliability Engineering \& System Safety. 2018; 172: p. 45-57.

29. Valls Miro J, Ulapane N, Shi L, Hunt D, Behrens M. Robotic pipeline wall thickness evaluation for dense nondestructive testing inspection. Journal of Field Robotics. 2018; 35(8): p. 1293-1310.

30. Svensson A, Solin A, Särkkä S, Schön T. Computationally efficient Bayesian learning of Gaussian process state space models. In ; 2016: Artificial Intelligence and Statistics (pp. 213-221).

31. Murphy KP. Conjugate Bayesian analysis of the Gaussian distribution. Vancouver:; 2007.

32. Di Maio F, Hoseyni SM, Zio E. Stima adattativa del rischio di rottura di componenti in pressione soggetti a creep con un approccio probabilistico. In ; 2018; Bologna: SAFAP 2018-Sicurezza ed affidabilità delle attrezzature a pressione.

33. ISPESL. Technical Procedure Concerning Calculation and Tests to Act on Creep-Operated Pressure Vessel Components Circular N.48/2003, technical procedure issued in Italian. Italy:; 2003.

34. NIMS. NIMS Atlas of creep deformation property. No. D-1, Creep deformation properties of 9Cr1MoVNb steel for boiler and heat exchangers. Tokyo:; 2007.

35. Storesund J, Samuelson L, Klasen B. Creep life assessment of pipe girth weld repairs with recommendations. OMMI. 2002; 1(3). 